\title{
La Unión Europea ante el desafío de la diversidad cultural. La implementación de la Convención de la UNESCO en la Unión Europea, diez años tras su entrada en vigor*,**
}

\author{
The European Union facing the Challenge of Cultural Diversity. \\ The Implementation of the UNESCO Convention in the \\ European Union, Ten Years After its Entry into Force
}

\author{
Belén Becerril Atienza \\ Universidad CEU San Pablo \\ becati@ceu.es
}

doi: http://dx.doi.org/10.18543/ced-57-2017pp69-100

\begin{abstract}
Sumario: I. Introducción.-II. Aproximación a la Convención sobre la Protección y la Promoción de la Diversidad de las Expresiones Culturales. 1. El contexto y los objetivos de la Convención. 2. Las obligaciones de las Partes y la relación de la Convención con otros instrumentos internacionales.-III. La implementación de la Convención en la política cultural de la Unión Europea. 1. El marco político y regulatorio de la política cultural. 2. Los programas de apoyo al sector cultural y creativo. 3 . La regulación de los servicios audiovisuales.-IV. Conclusiones.
\end{abstract}

Resumen: En el mes de marzo de 2017 se han cumplido diez años de la entrada en vigor de la Convención de la UNESCO sobre la Protección y la Promoción de la Diversidad de las Expresiones Culturales, un instrumento normativo internacional que la Unión Europea había promovido, negociado por vez primera en el seno de la UNESCO y ratificado. Es por tanto un buen momento para valorar en qué medida la Unión ha contribuido a la protección y promoción de la diversidad cultural en este período y, más allá de la retórica, se ha convertido efectivamente en el relevante valedor de la Convención que se anunciaba en 2007. Con este fin, este artículo aborda inicialmente la Convención, para recordar el contexto de su aprobación y sus objetivos, y señalar en particular las obligaciones que de la misma se derivan para las Partes, así como su relación con otros instrumentos internacionales. Se examina a continuación la contribución

* Recibido el 12 de enero de 2017, aceptado el 11 de mayo de 2017.

** El presente estudio se enmarca dentro de la Red de Excelencia sobre «Los actuales desafíos del Derecho Internacional», del Plan Estatal de Investigación Científica y Técnica y de Innovación 2013-2016 (DER15-69273-RED). 
de la Unión Europea a su implementación en su dimensión interna. Para ello se valora en qué medida se ha tenido en cuenta el espíritu y la letra de la Convención desde el año 2007 en el marco político regulatorio de la política cultural, en los programas de apoyo al sector cultural y creativo y en la regulación de los servicios audiovisuales.

Palabras clave: Convención Diversidad Cultural, UNESCO, Unión Europea.

Abstract: In March 2017, ten years have passed since the entry into force of the UNESCO Convention on the Protection and Promotion of the Diversity of Cultural Expressions, an international normative instrument promoted, negotiated and ratified by the European Union. It is therefore a good time to assess to what extent the Union has contributed to the protection and promotion of cultural diversity in this period and whether, beyond rhetoric, it has indeed become the relevant supporter of the Convention announced in 2007. To this end, this article initially addresses the Convention, in order to recall the context of its adoption and its objectives, and to examine in particular the obligations arising for the Parties, as well as its relationship with other international instruments. This paper examines the contribution of the European Union to the implementation of the Convention in its internal dimension. To that end, it assesses the extent to which the spirit and the letter of the Convention have been taken into account since 2007 in the regulatory framework of the European cultural policy, in the support programs for the cultural sector, and in the regulation of audiovisual services.

Keywords: Convention on Cultural Diversity, UNESCO, European Union.

\section{Introducción}

En octubre de 2005 la 33. ${ }^{\text {a }}$ Conferencia General de la Organización de las Naciones Unidas para la Educación, la Ciencia y la Cultura (UNESCO) aprobaba la Convención sobre la Protección y la Promoción de la Diversidad de las Expresiones Culturales ${ }^{1}$ (en adelante, la Convención de la Diversidad Cultural o simplemente, la Convención). Culminaba así un proceso que se había iniciado en 2001 con la aprobación de la Declaración Universal de la Diversidad Cultural ${ }^{2}$, y que la UNESCO se había comprome-

${ }^{1}$ Convención sobre la protección y la promoción de la diversidad de las expresiones culturales, París, 20 de octubre de 2005, acceso el 10 de noviembre de 2016:

http://portal.unesco.org/es/ev.php-URL_ID=31038\&URL_DO=DO_TOPIC\&URL_ SECTION=201.html.

2 Declaración Universal de la UNESCO sobre la Diversidad Cultural, 2 de noviembre de 2001, acceso el 10 de noviembre de 2016:

http://portal.unesco.org/es/ev.php-URL_ID=13179\&URL_DO=DO_TOPIC\&URL_ SECTION=201.html. 
tido a llevar a término mediante la aprobación de una Convención ${ }^{3}$. Tras dos años de negociaciones, la Convención de la Diversidad Cultural recibía un amplísimo apoyo de la comunidad internacional, con el voto favorable de 148 Estados, cuatro abstenciones - Australia, Honduras, Nicaragua y Liberia $-\mathrm{y}$ los votos en contra de Estados Unidos e Israel. Tras un rápido proceso de ratificación ${ }^{4}$, la nueva Convención entraba en vigor en marzo de 2007.

La Unión Europea impulsó la idea de aprobar un instrumento normativo internacional sobre la diversidad cultural, señalando la necesidad de establecer un pilar cultural en la gobernanza internacional ${ }^{5}$. A continuación, la Unión participó de modo novedoso en las negociaciones de la UNESCO. Por vez primera, su Consejo Ejecutivo invitaba con carácter excepcional a la Comunidad Europea a participar activamente en los trabajos de la Convención ${ }^{6}$, a pesar de gozar formalmente tan sólo de un estatuto de observador, que normalmente implica una restringida participación en las reuniones. La UNESCO respondía así a la solicitud de los Estados miembros de la Unión que, siguiendo la recomendación de la Comisión ${ }^{7}$, habían reclamado para la Comunidad un rol activo en las negociaciones, considerando el impacto directo que algunas disposiciones de la Convención tendrían en sus competencias.

La Comunidad obtenía así, con cierto retraso debido a las reticencias iniciales en la UNESCO, un estatuto de observador reforzado, similar al de los miembros salvo en lo que a la exclusión del derecho de voto se refiere. La Comisión participaría en las negociaciones, en nombre de la Comunidad, junto con los Estados miembros, de acuerdo con la distribución de responsabilidades recogida en un código de conducta que determinaba cuidadosamente los cometidos de la Presidencia y los

${ }^{3}$ Resolución 32 C/34, UNESCO, aprobada, previo informe de la Comisión IV, en la 21. a sesión plenaria, París, 17 de octubre de 2003: 68.

${ }^{4}$ La Convención ha sido ratificada por 143 Estados así como por la Unión Europea. El listado puede consultarse en la página web de la UNESCO, acceso el 10 de noviembre de 2016:

http://www.unesco.org/eri/la/convention.asp?order=alpha\&language=S\&KO=31038.

5 Comunicación de la Comisión al Consejo y al Parlamento Europeo «Hacia un instrumento internacional sobre la Diversidad Cultural», COM (2003) 520 final, 27 de agosto de 2003.

${ }^{6}$ Decisión 171 EX/60, UNESCO, Decisiones adoptadas por el Consejo Ejecutivo en su 171. a reunión (2-28 de abril de 2005), París, 25 de mayo de 2005: 78.

7 Recomendación de la Comisión al Consejo para que autorice a la Comisión a participar, en nombre de la Comunidad, en las negociaciones de la UNESCO en relación con la Convención sobre la Protección de la Diversidad de los Contenidos Culturales y las Expresiones Artísticas, SEC (2004) 1062 final, 1 de septiembre de 2004. 
de la Comisión ${ }^{8}$. Como resultado, la Comunidad Europea adquirió en las negociaciones una visibilidad inusual ${ }^{9}$. En esta ocasión, logró hablar con una sola voz, y en parte como consecuencia de este hecho, la mayor parte de sus propuestas serían incorporadas al texto final ${ }^{10}$. La Comisión se congratulaba, al término del proceso, de «la unidad ejemplar de la Comunidad Europea, que le permitió actuar como uno de los motores de las negociaciones» ${ }^{11}$.

Esta Convención de la UNESCO fue también la primera de esta organización en contener una disposición que permitía la adhesión de organizaciones de integración económica regional (art. 27). En virtud de la misma, la Unión Europea depositaba, el 18 de diciembre de 2006, su instrumento de adhesión ${ }^{12}$, acordándose que en aquellos ámbitos que fuesen de competencia exclusiva de la Comunidad, la Comisión la representaría en los órganos creados por la Convención, en particular, en la Conferencia de las Partes. Se informaba también ${ }^{13}$ sobre las responsabilidades respectivas de los Estados y la Comunidad en el marco de la Convención, destacándose las competencias de la Comunidad en lo que respecta a la política comercial común, la política de cooperación al desarrollo, la política de cooperación con los países industrializados, la libre circulación de bienes, personas, servicios y capitales, la competencia y el mercado interior, incluida la propiedad intelectual, así como la obligación de tener en cuenta los aspectos culturales en su actuación en virtud de otras disposiciones del Tratado.

A partir de ese momento la Convención se consolidó como un elemento primordial en la formulación de la política cultural de la Unión. El Consejo manifestó su apoyo señalando que la Convención constituía «un pilar perti-

${ }^{8}$ Consejo de la UE, Proyecto de código de conducta entre el Consejo, los Estados miembros y la Comisión, relativo a las negociaciones en el marco de la UNESCO de una Convención internacional sobre la protección de la diversidad de los contenidos culturales y las expresiones artísticas, doc. 5518/05, 28 de enero de 2005. Véase también Delia Ferri, «EU Participation in the UNESCO Convention on the Protection and Promotion of the Diversity of Cultural Expressions: Some Constitutional Remarks», European Diversity and Autonomy Papers, n. $^{\circ} 3$ (2005): 1-34.

${ }^{9}$ Lucia Cavicchioli, «The European Community at UNESCO: an exceptionally active observer?», en The United Nations and the European Union: an ever stronger partnership, ed. por Jan Wouters, Frank Hoffmeister y Tom Ruys (The Hague: Asser, 2006): 136.

${ }^{10}$ Evangelia Psychogiopoulou, Integration of Cultural Considerations in European Union Law and Policies (Leiden: Martinus Nijhoff Publishers, 2007): 120.

${ }^{11}$ Comisión Europea: IP/05/676, Bruselas, 6 de junio de 2005.

12 El 18 de mayo de 2006 el Consejo adoptaba la decisión relativa a su celebración, declarando aprobada, en nombre de la Comunidad, la Convención y autorizando al presidente del Consejo a designar a la persona habilitada para depositar el instrumento de adhesión ante la UNESCO. Decisión n. ${ }^{\circ}$ 515/2006/CE del Consejo de 18 de mayo de 2006.

13 Véase, en el anexo 1.b) de dicha Decisión, la Declaración de la Comunidad Europea en aplicación del artículo 27, apartado 3, letra c) de la Convención. 
nente y eficaz para la promoción de la diversidad cultural» ${ }^{14}$. La Comisión, que precisamente en 2007 realizaba el ejercicio de reflexión más sistemático y ambicioso emprendido hasta entonces sobre la relación de la Unión Europea con la cultura y sus prioridades para el futuro, otorgaría a esta Convención de la UNESCO un lugar destacado en el documento resultante, la Agenda Europea para la Cultura ${ }^{15}$.

La nueva Convención entraba en vigor en marzo de 2007, hace ahora diez años. Es por tanto un buen momento para valorar en qué medida la Unión ha contribuido, más allá de su formulación, a la protección y promoción de la diversidad cultural en este período. Para ello, se abordará en primer lugar la Convención, con el fin de recordar el contexto de su aprobación y sus objetivos, y señalar en particular las obligaciones que de la misma se derivan para las Partes así como su relación con otros instrumentos internacionales. Seguidamente, se examinará la contribución de la Unión Europea a la implementación de la Convención en su dimensión interna. Se analizará en qué medida se ha tenido en cuenta el espíritu y la letra de la Convención desde el año 2007 en el marco político y regulatorio de la política cultural, en los programas de apoyo al sector cultural y creativo, y en la regulación de los servicios audiovisuales. Con este fin se realizará un análisis de los actos aprobados en la materia por las instituciones europeas, así como un estudio crítico de la literatura académica.

Es preciso notar que se excluyen de este análisis otras políticas internas europeas con una dimensión cultural. También, las medidas adoptadas por la Unión Europea para implementar la Convención en su dimensión externa, como los programas culturales de cooperación internacional, que se han fortalecido desde $2007^{16}$, o la firma de protocolos culturales anejos a los acuerdos comerciales, una práctica iniciada en 2008. Conviene al menos mencionar la novedad de estos protocolos, con los que la Unión quería situarse en primera línea en la implementación de la Convención y promover los intercambios culturales en particular en favor de los países en desarro-

14 Decisión n. ${ }^{\circ}$ 515/2006/CE del Consejo de 18 de mayo de 2006.

15 Comunicación de la Comisión relativa a una Agenda Europea para la Cultura en un Mundo en vías de Globalización, COM (2007) 242 final, de 10 de mayo de 2007. Véase también la Resolución del Consejo relativa a una Agenda Europea para la Cultura, de 16 de noviembre de 2007 (2007/C 287/01).

16 Carmina Crusafón concluye del estudio de los casos de América Latina y el Mediterráneo que la dimensión cultural está crecientemente presente en las relaciones externas de la Unión desde la firma de la Convención, aunque señala que es demasiado pronto para concluir si ello resulta en una verdadera promoción cultural. «EU Cultural Cooperation with third countries: The Cases of Latin America and the Mediterranean», en Cultural Governance and the European Union: Protecting and Promoting Cultural Diversity in Europe, ed. por Evangelia Psychogiopoulou (Palgrave Macmillan, 2015): 235. 
$1 \mathrm{lo}^{17}$. Constituyen pues una manifestación relevante del esfuerzo por implementar la Convención, si bien han resultado particularmente controvertidos y sus efectos han sido limitados pues sólo afectan a un pequeño número de Estados y sólo alteran marginalmente el status quo ${ }^{18}$.

El presente estudio se limita pues a la implementación de la Convención por parte de la Unión en su dimensión interna. Ello se debe a que, dada la vaguedad y la ausencia de carácter coactivo de la mayor parte de las disposiciones de la Convención, su implementación dependerá en gran medida de la voluntad de las Partes y en particular, de las medidas adoptadas para su implementación dentro de sus fronteras. Por ello se ha señalado que, habiendo sido la Unión Europea un actor clave en las negociaciones, y habiendo hecho de la promoción de la diversidad cultural y de la Convención una prioridad de su política cultural, el modelo de implementación de la Unión podría constituir un ejemplo relevante para la comunidad internacional ${ }^{19}$. Cabe también mencionar que la Unión tiene una larga experiencia en la búsqueda de un equilibrio entre el principio de libre circulación de bienes y servicios culturales y el respeto a las políticas culturales nacionales, experiencia que podría resultar útil en el camino emprendido por la Convención para atender a los aspectos culturales en la gobernanza global.

\section{Aproximación a la Convención sobre la Protección y la Promoción de la Diversidad de las Expresiones Culturales}

\section{El contexto y los objetivos de la Convención}

La tensión entre el comercio internacional y la diversidad cultural, a la que frecuentemente se hace referencia con los términos «comercio versus cultura», late desde su origen tras la firma de la Convención de la Diversi-

17 Comisión Europea, Argumentaire on the Title on Cultural Cooperation in future EU trade agreements, Bruselas, mayo de 2007, acceso el 10 noviembre de 2016:

http://trade.ec.europa.eu/doclib/docs/2007/may/tradoc_134655.pdf

18 Jan Loisen, «Mainstreaming Culture in EU External Relations through Protocols on Cultural Cooperation: Fostering or Faltering Cutural Diversity?», en The Palgrave Handbook of European Media Policy, ed. por Karen Donders, Caroline Pawels y Jan Loisen (Palgrave Macmillan, 2014): 522. Véase también: Jordi Baltà, «Aplicación en la Unión Europea de la Convención de la UNESCO de 2005 sobre la Protección y Promoción de la Diversidad de las Expresiones Culturales», solicitado por la Comisión de Cultura y Educación del Parlamento Europeo: http://www.diversitystudy.eu/ms/spanish_study_version_10_10_2010.pdf. Acceso 10 de noviembre 2016.

19 Mira Burri, «Business as Usual? An Appraisal of the Implementation of the UNESCO Convention on Cultural Diversity into EU's Media Law and Policies», European Law Review n. 3 (2003): 803 . 
dad Cultural. Si bien ésta se refiere de una manera general a la diversidad de las expresiones culturales, su objetivo fue desde el inicio responder a una preocupación relativa al desigual intercambio internacional de bienes y servicios culturales, y de manera muy particular, los de carácter audiovisual $^{20}$. Esta cuestión había sido objeto de desencuentros entre la Unión Europea y los Estados Unidos desde mediados de los años ochenta. En las negociaciones de la Ronda de Uruguay, los Estados Unidos habían propuesto la inserción del sector cultural, incluido el audiovisual, en el proceso liberalizador, de modo que recibiese el mismo tratamiento que los demás servicios. La Comunidad Europea solicitó en cambio que la cultura fuera incluida como una excepción general, equiparándola a otros intereses generales como la seguridad o el orden público. El acuerdo de Marrakech llegó a una solución de compromiso: la cultura no quedaba recogida como una excepción general, pero en la práctica el audiovisual no se liberalizaría pues pocos Estados asumirían compromiso alguno en este sector. No obstante, la dura confrontación habida explica que, finalizada la Ronda, algunos Estados como Francia y Canadá planteasen la necesidad de establecer un instrumento internacional que abordarse esta cuestión desde otra perspectiva y en otro foro ${ }^{21}$.

Los trabajos comenzaron en la UNESCO en el año 2003. Los miembros acordaron centrar su acción normativa en «La protección de la diversidad de los contenidos culturales y de la expresión artística», con el fin de proteger «la diversidad de los contenidos culturales y de las formas de expresión artística vehiculadas por la industria de la cultura, aspectos que parecen correr especial peligro en el contexto actual de mundialización» ${ }^{22}$. En aquel momento, ya se habían aprobado las dos declaraciones que constituirían los antecedentes más directos de la Convención: la Declaración sobre la Diversidad Cultural del Consejo de Europa, del año $2000^{23}$ y la mencionada Declaración Universal de la Diversidad Cultural de la UNESCO, de 2001.

${ }^{20} \mathrm{El}$ valor total de las exportaciones de servicios culturales a nivel mundial en 2012 fue de 128.5 mil millones de dólares. El porcentaje de los países en desarrollo representa un 1.6\%. El 52,4\% corresponden a los Estados Unidos de América. Datos del Instituto de Estadística de la UNESCO recogidos en el informe Repensar las Políticas Culturales, UNESCO, 2015: 121-133.

${ }^{21}$ Carol Balassa, «The Impact of the US Position in the Trade and Culture Debate», The UNESCO Convention on the Diversity of Cultural Expressions, ed. por Toshiyuki Kono y Steven Van Uystel (Cambridge: Intersentia, 2012): 84.

$22166 \mathrm{EX} / 28$ «Estudio preliminar de los aspectos técnicos y jurídicos relacionados con la conveniencia de elaborar un instrumento normativo sobre la diversidad cultural» de 12 de marzo de 2003: 5-7.

23 Adoptada por el Comité de Ministros el 7 de diciembre del 2000 en el 733. ${ }^{\circ}$ encuentro de Ministros. 
La primera de ellas constituía una manifestación temprana del nuevo énfasis en el concepto de diversidad cultural y del particular interés de los países europeos por esta cuestión. La declaración reconocía que «el respeto por las diversidades culturales es una condición esencial de la sociedad humana» y afirmaba que el desarrollo de las nuevas tecnologías de información, la globalización y el comercio multilateral tienen un impacto sobre la diversidad cultural. Disponía por ello, que «las políticas culturales y audiovisuales, que promueven el respeto de la diversidad cultural, son un complemento necesario a las políticas comerciales» (art.2.). La Declaración manifestaba a su vez la centralidad del sector audiovisual, al que se refería repetidamente, en este debate.

La Declaración Universal de la Diversidad Cultural de la UNESCO de 2001 reconocía, por vez primera, que la diversidad cultural «constituye el patrimonio común de la humanidad» y «debe ser reconocida y consolidada en beneficio de las generaciones presentes y futuras» (art.1). También se refería a tres cuestiones que, como se verá, cobrarían especial relevancia en la posterior Convención: en primer lugar, el carácter específico de los bienes y servicios culturales «que, por ser portadores de identidad, de valores y sentido, no deben ser considerados mercancías o bienes de consumo como los demás» (art.8); en segundo lugar, ante los desequilibrios en los flujos e intercambios a escala mundial, se señalaba la necesidad de «reforzar la cooperación y la solidaridad internacionales para que todos los países, especialmente los países en desarrollo y los países en transición, puedan crear industrias culturales viables y competitivas» (art.10); y en tercer lugar, se enfatizaba el papel de las políticas públicas: «las fuerzas de mercado por sí solas no pueden garantizar la preservación y promoción de la diversidad cultural, claves para un desarrollo humano sostenible. Desde este punto de vista, se debe reafirmar la preeminencia de las políticas públicas, en colaboración con el sector privado y la sociedad civil» (art.11).

Si bien la declaración de la UNESCO, al igual que la del Consejo de Europa, carecía de carácter vinculante, ambas eran expresión de la creciente relevancia de esta cuestión. Como señalaba la Comisión Europea en 2003, la diversidad cultural se había convertido en una de las principales cuestiones debatidas a escala internacional entre las organizaciones internacionales $\mathrm{y}$ regionales ${ }^{24}$.

Ambas declaraciones manifestaban también la consolidación del concepto mismo de diversidad cultural. La preocupación por el desequilibrio en los intercambios internacionales de bienes y servicios culturales venía

${ }^{24}$ Comunicación de la Comisión «Hacia un instrumento internacional sobre la Diversidad Cultural»: 2. 
de muy lejos, habiéndose manifestado en los años ochenta tanto en las negociaciones en la Ronda de Uruguay como en el seno de la UNESCO. En esta organización, la iniciativa «New World Information and Communication Order» y el informe MacBride ${ }^{25}$ generaron una tensión que explica el abandono temporal de la organización por parte de los Estados Unidos y el Reino Unido. Sin embargo, tanto en la UNESCO como en la Organización Mundial de Comercio (en adelante, OMC) hasta el nuevo siglo, la resistencia a avanzar en la liberalización de bienes y servicios culturales se realizaba en nombre de la controvertida excepción cultural $^{26}$. Tras la aprobación de las Declaraciones del Consejo de Europa y de la UNESCO, un nuevo concepto de diversidad cultural, más complejo y abierto, se consolidaba. Un concepto que incide en la necesidad de preservar cierto equilibrio en los intercambios y reivindica el derecho de los Estados a adoptar políticas con ese fin, pero que también enfatiza que la diversidad cultural se fortalece mediante la circulación de ideas e intercambios ${ }^{27}$.

Con estos antecedentes, la nueva Convención de la Diversidad Cultural aprobada en 2005 partía de la afirmación de que la diversidad cultural es una característica esencial de la humanidad, un patrimonio común que debe valorarse y preservarse. A continuación, recogía un primer objetivo de carácter general consistente en proteger y promover la diversidad de las expresiones culturales. A este se sumaban ocho objetivos más concretos, seguidos de ocho principios rectores, que se referían a la creación de condiciones para que las culturas puedan prosperar, al fomento del diálogo entre culturas a fin de garantizar intercambios más equilibrados, al fomento de la interculturalidad, a la importancia del vínculo existente entre la cultura y el desarrollo... Cabe destacar en particular los tres últimos objetivos, que venían a confirmar y fortalecer, en unos términos más precisos, unas ideas que estaban ya presentes en Declaración de la UNESCO sobre la Diversidad Cultural:

- Reconocer la índole específica de las actividades y los bienes y servicios culturales en su calidad de portadores de identidad, valores y significado;

- Reiterar los derechos soberanos de los Estados a conservar, adoptar y aplicar las políticas y medidas que estimen necesarias para proteger y promover la diversidad de las expresiones culturales en sus respectivos territorios;

25 Informe Mac Bride, Un solo Mundo, voces múltiples, puede consultarse en su edición en español del Fondo de Cultura Económica, México, 1980.

${ }^{26}$ Una buena recopilación de los argumentos de los partidarios de la excepción cultural puede consultarse en Serge Regourd, L'exception culturelle (París: Puf, 2003).

27 Sobre el concepto de diversidad cultural: Jesús Prieto de Pedro, Excepción y diversidad cultural (Madrid: Alternativas, 2005): 29. 
- Fortalecer la cooperación y solidaridad internacionales en un espíritu de colaboración, a fin de reforzar, en particular, las capacidades de los países en desarrollo.

Estos fueron de hecho los tres elementos que la Comunidad acentuó en las negociaciones ${ }^{28}$, junto con la adecuada articulación del Convenio con otros instrumentos internacionales y el énfasis en los derechos humanos y las libertades fundamentales que quedaría recogido en su artículo segundo, en virtud del cual nadie podrá invocar las disposiciones de la Convención para atentar contra los mismos. La inclusión de los objetivos mencionados en la versión final de la Convención explica la satisfacción expresada por la Comisión al término del proceso $^{29}$.

\section{Las obligaciones de las Partes y la relación de la Convención con otros instrumentos internacionales}

Si bien la sección IV de la Convención se refiere a los derechos y obligaciones, un análisis detallado del texto pone de manifiesto la parquedad de las obligaciones allí recogidas. Bien al contrario, a lo largo del texto parece primar el objetivo de reiterar «los derechos soberanos de los Estados a conservar, adoptar y aplicar las políticas y medidas que estimen necesarias para proteger y promover la diversidad de las expresiones culturales en sus respectivos territorios» (art.1.h). Este es en efecto, un objetivo expresado hasta la saciedad pues se vuelve a recoger en forma de principio rector (art. 2.2.), de norma general relativa a los derechos y obligaciones (art. 5.1.), y aún de nuevo (art. 6.1.) bajo el epígrafe «Derechos de las Partes en el plano nacional».

La Convención enfatiza los derechos por encima de las obligaciones ${ }^{30}$. El artículo 6.2. contiene un listado abierto de medidas que podrán ser adop-

${ }^{28}$ Communication of the European Community and its Member States to UNESCO on the preliminary draft UNESCO Convention on the protection of the diversity of cultural contents and artistic expressions,

Paris, 15 noviembre 2004. Puede consultarse en la siguiente página web, acceso el 10 de noviembre de 2016: http://trade.ec.europa.eu/doclib/docs/2004/december/tradoc_120449.pdf.

${ }^{29}$ La nota de prensa enfatizaba precisamente los tres objetivos señalados. Comisión Europea: IP/05/676.

${ }^{30}$ Evangelina Psychogiopoulou, «The Convention on the Diversity of Cultural Expressions and the European Union», en The UNESCO Convention..., editado por Toshiyuki Kono y Steven Van Uystel: 367. En el mismo sentido, Mira Burri, «Business as Usual? An Appraisal of the Implementation of the UNESCO Convention on Cultural Diversity into EU's Media Law and Policies»: 804; Rachael Craufurd Smith, «The UNESCO Convention on the Protection and Promotion of the Diversity of Cultural Expressions: Building a New World Information and Communication Order?», International Journal of Communication, n. ${ }^{\circ} 1$ (2007): 37. 
tadas por las Partes, pero no impone obligación alguna. Entre las mencionadas se recogen medidas reglamentarias encaminadas a la protección y promoción de la diversidad, medidas para conceder asistencia financiera pública, para crear y apoyar las instituciones de servicio público... Se citan tanto medidas de promoción, que previsiblemente adoptarán la forma de ayudas y subsidios, como medidas de protección, de carácter más controvertido y más susceptibles de incompatibilidad con las normas de la OMC. Conviene señalar que el artículo 6 no precisa en modo alguno los límites de tales medidas, ni se refiere a los principios de eficacia o proporcionalidad en su aplicación.

El artículo 7 recoge, bajo el epígrafe «Medidas para promover las expresiones culturales», que las Partes procurarán crear un entorno que incite a las personas y a los grupos a crear, producir, difundir y distribuir sus propias expresiones culturales y tener acceso a ellas. También dispone que las Partes procurarán que se reconozca la contribución de artistas y personas que participan en los procesos creativos. En ambos casos el uso verbo procurar descarta obligatoriedad alguna. En la misma línea, el artículo 8, sobre las «Medidas para proteger las expresiones culturales», dispone que una Parte podrá determinar si hay situaciones especiales en que las expresiones culturales en su territorio corran riesgo de extinción o sean objeto de una grave amenaza. De nuevo, dispone que los Estados podrán entonces adoptar cuantas medidas consideren necesarias para proteger y preservar las expresiones culturales.

En las siguientes disposiciones la Convención utiliza unos términos más coactivos en relación con algunas obligaciones que podríamos denominar auxiliares ${ }^{31}$. El artículo 9 establece que las partes proporcionarán cada cuatro años a la UNESCO información apropiada acerca de las medidas que hayan adoptado. El artículo 10 establece que las Partes deberán promover el entendimiento de la importancia que revisten la protección y fomento de la diversidad mediante programas de educación y sensibilización. El artículo 11 dispone que las Partes fomentarán la participación activa de la sociedad civil. Alguna obligatoriedad parece también desprenderse de las disposiciones que siguen, referidas a la cooperación internacional. El artículo 16 dispone que los países desarrollados facilitarán un trato preferente a los trabajadores, bienes y servicios culturales de los países en desarrollo y el 17 establece que las Partes se prestarán asistencia mutua en situaciones de grave peligro para las expresiones culturales cuya naturaleza, no obstante, no se precisa.

31 Ivan Bernier, «The Convention on the Protection and the Promotion of the Diversity of Cultural Expressions, a Cultural Analysis», en The UNESCO Convention... ed. por Toshiyuki Kono y Steven Van Uystel: 106. 
Pocas obligaciones concretas parecen pues desprenderse de esta Convención, lo que parece confirmar la idea de que el objetivo primordial de sus promotores fue más bien afirmar el derecho de las Partes a desarrollar sus propias políticas culturales, es decir, conceder a los Estados más espacio político y legitimidad para desarrollar tales políticas, y de este modo, contrarrestar la presión a favor de la liberalización de servicios audiovisuales que tiene lugar en el marco del comercio internacional. Esta cuestión invita pues a plantearse uno de los asuntos que fueron más controvertidos en las negociaciones: la relación de las disposiciones de la Convención con otros instrumentos internacionales. El artículo 20 establece a este respecto los principios de potenciación mutua, complementariedad y no subordinación. Las Partes disponen que sin subordinar esta Convención a los demás tratados, la tendrán en cuenta cuando los interpreten y apliquen y fomentarán su potenciación mutua (art.20.1). No obstante, se establece también que «ninguna disposición de la presente Convención podrá interpretarse como una modificación de los derechos y obligaciones de las Partes que emanen de otros tratados internacionales en los que sean parte» (art.20.2).

Esto significa que, a pesar de ese énfasis tan pronunciado en el derecho de las Partes a desarrollar sus políticas culturales, nada en la Convención podrá impedir que las medidas adoptadas en virtud del mencionado artículo 6 con el fin de proteger y promover la diversidad de las expresiones culturales sean consideradas incompatibles con otros instrumentos internacionales, y muy en particular con los Acuerdos de la OMC. En efecto, esto quedó fuera de toda duda tras la decisión de su Órgano de Apelación en el asunto China - Publicaciones y productos audiovisuales de esparcimiento ${ }^{32}$. En este contexto, no es de extrañar que se haya señalado la debilidad de la respuesta de la Convención al principal motivo que llevó a su negociación ${ }^{33}$. Esta limitación, junto con la ausencia de un mecanismo de solución de conflictos que supere la mera conciliación, explica que algunos se hayan planteado si después de todo, el resultado de la Convención no ha consistido en poco más que sensibilizar sobre la diversidad de las expresiones culturales $\mathrm{y}$ aportar un glosario útil para futuros debates ${ }^{34}$.

${ }^{32}$ Informe del Órgano de Apelación, China - Medidas que afectan a los derechos comerciales y los servicios de distribución respecto de determinadas publicaciones y productos audiovisuales de esparcimiento, WT/ DS36321/AB/R, 21 de diciembre 2009.

${ }^{33}$ Lilian Richieri Hanania (ed.), Cultural Diversity in International Law, The effectiveness of the UNESCO Convention on the Protection and Promotion of the Diversity of Cultural Expressions (Abingdon: Routledge, 2014): 299.

${ }^{34}$ Rostam J. Neuwirth, «The Convention on the Diversity of Cultural Expressions. A critical Analysis of the provisions», The UNESCO Convention..., ed. por Toshiyuki Kono y Steven Van Uystel: 69. 
En todo caso conviene considerar que, dado el apoyo casi unánime que recabó, la Convención tiene un notable valor político y fortalece la posición de los Estados a la hora de resistir la liberalización de servicios culturales en la $\mathrm{OMC}^{35}$. Además, si bien no pondrá en cuestión las obligaciones derivadas de las disposiciones de otros Tratados, puede ejercer un efecto indirecto y afectar su interpretación pues, como se ha señalado, las Partes deberán tener en cuenta la Convención cuando interpreten y apliquen los demás tratados. También los órganos de resolución de conflictos de la OMC deberán interpretar los Tratados teniendo en cuenta las normas relevantes de derecho internacional aplicables en las relaciones entre las Partes, tal y como requiere el artículo 31.3 c) de la Convención de Viena $^{36}$.

Por último, en lo que respecta a la relación de la Convención con el ordenamiento jurídico de la Unión Europea, esta formuló una Declaración unilateral en virtud de la cual los Estados miembros «aplicarán en sus relaciones mutuas las disposiciones de la Convención de conformidad con las normas internas comunitarias y sin perjuicio de las modificaciones que, según convenga, se hagan de esas normas» ${ }^{37}$. Esta cláusula se asemeja a las denominadas «cláusulas de desconexión» introducidas por los Estados miembros en la práctica del derecho internacional con el fin de asegurar la posibilidad de respetar las obligaciones impuestas por el derecho de la Unión Europea al implementar sus obligaciones internacionales $^{38}$. No obstante, como se verá más adelante, en el asunto UTECA ${ }^{39}$ el Tribunal de Justicia ha sugerido que tomará en consideración la Convención, en el sentido del art.20.1., a la hora de aplicar el Derecho de la Unión. Una posición bien distinta a la que se puso de manifiesto en la $\mathrm{OMC}$ en el mencionado asunto China - Publicaciones y productos audiovisuales de esparcimiento, en el que, en la lógica del art.20.2., se descartó la aplicación de la Convención.

35 Así se considera que efectivamente ha ocurrido en: Richieri Hanania, Cultural Diversity...: 299.

${ }^{36}$ Rachael Craufurd Smith, «The UNESCO Convention on the Protection and Promotion of the Diversity of Cultural Expressions: Building a New World Information and Communication Order?»: 45-52.

37 Véase la Declaración unilateral en nombre de la Comunidad en relación con el depósito del instrumento de adhesión en el anexo 2 de la Decisión n. ${ }^{\circ}$ 515/2006/CE del Consejo.

38 Magdalena Licková, «The CDCE in the European Union - a mixed agreement and its judicial application», en Cultural Diversity in International Law..., ed. por Lilian Richieri Hanania: 229.

39 Sentencia de 5.3.2009, UTECA, asunto C-222/07. 


\section{La implementación de la Convención en la política cultural de la Unión Europea}

La relación de la Unión Europea con la cultura es un asunto especialmente sensible y complejo, debido en parte a la confusión que rodea al polisémico término cultura y a la relación de la misma con las diversas dimensiones y políticas de la Unión. Desde los años cincuenta, cuando los Tratados apenas mencionaban la cultura ${ }^{40}$, hasta nuestros días, se ha recorrido un largo y difícil camino, al término del cual podríamos decir que la Unión dispone hoy de una política cultural ${ }^{41}$.

En dicho camino destacan dos momentos cruciales. El primero, 1992, vino marcado por la inclusión en el Tratado de Maastricht de la nueva disposición cultural (hoy, art. 167 TFUE). Se formalizaba así una acción cultural que se había desarrollado de manera dispersa pero creciente, envuelta en cierta nebulosa jurídica. Una acción que inicialmente se centraba en los aspectos económicos de la cultura, más estrechamente vinculados al mercado interior, y en la protección del patrimonio, pero que progresivamente fue abarcando otras materias como la promoción de la industria audiovisual, del libro y la lectura, y otras formas de expresión cultural. El segundo momento, 2007, vino marcado por la aprobación de la Agenda Europea para la Cultura. Esto se produjo tras la aprobación de la Convención de la UNESCO y poco antes de su entrada en vigor. Con la Agenda, la Unión daba un gran paso en su discurso programático, aportando mayor orientación y coherencia a lo que en el pasado había sido poco más que una serie de iniciativas aisladas, una acción cultural más que una verdadera política.

A lo largo de este camino se ha mantenido una cierta continuidad. Ninguno de los dos momentos señalados significó una total reordenación de las prioridades y los programas, sino que más bien cada paso se construyó sobre el anterior. La Convención de la UNESCO no rompió tampoco esa continuidad. Por ello, para valorar en qué media la Unión ha implementado la Convención es necesario contemplar algunos elementos que se habían desarrollado anteriormente en su acción cultural. Así lo hacía de hecho la Comisión en su informe relativo a la implementación de la Convención de $2012^{42}$.

40 Tan sólo el art. 131TCEE (hoy, 198 TFUE), sobre la asociación de los países y territorios de ultramar.

${ }^{41}$ Belén Becerril Atienza, Hacia una política Cultural de la Unión Europea (Cizur Menor: Aranzadi, 2015): 386.

${ }^{42}$ Commission Staff Working document, Quadrennial Report on behalf of the European Union on Measures to Project and Promote the Diversity of Cultural Expressions in the 
Por otra parte, conviene señalar la diversidad de las políticas de la Unión que de un modo u otro tienen un efecto en el sector cultural. Esta transversalidad queda subrayada en la llamada cláusula horizontal del artículo 167.4.TFUE, que precisa que «La Unión tendrá en cuenta los aspectos culturales en su actuación en virtud de otras disposiciones del presente Tratado en particular a fin de respetar y fomentar la diversidad de sus culturas». Esta cláusula constituyó una de las más relevantes novedades de esta disposición pues si bien, como señaló la Comisión, esa exigencia se había puesto en práctica a lo largo de la historia de la Comunidad, teniéndose en cuenta consideraciones culturales en el establecimiento de los actos comunitarios, con la cláusula horizontal esta exigencia se volvía «obligatoria y sistemática» ${ }^{43}$.

En su primer informe sobre la implementación de la Convención ${ }^{44}$, la Comisión recogía el conjunto de políticas europeas con un impacto en la diversidad de las expresiones culturales. Incluía así, tanto las medidas adoptadas específicamente para el sector cultural y audiovisual, como las medidas que afectan el entorno en el que operan estos sectores, como la sociedad de la información, el mercado interior - incluyendo los derechos de propiedad intelectual -, la política industrial y de innovación, el derecho de la competencia incluyendo lo relativo a las ayudas de estado, la fiscalidad, la política de cohesión, la política comercial común, la cooperación al desarrollo y la cooperación económica, financiera y técnicas con terceros Estados. Por la imposibilidad de abordar a continuación tan diversas políticas, limitaremos este estudio, en lo que a las políticas internas se refiere, a las medidas más relevantes adoptadas específicamente en el sector cultural y audiovisual.

\section{El marco político y regulatorio de la política cultural}

En primer lugar, cabe recordar que desde la entrada en vigor del Tratado de Maastricht, la disposición fundamental sobre la política cultural es el artículo 167 TFUE. En virtud de esta disposición, la cultura queda definida como un ámbito en el que la Unión puede llevar a cabo accio-

Framework of the 2005 UNESCO Convention, SWD (2012) 129 final, de 23 de febrero de 2012. Dicho informe acompaña al documento: Commission, Report on Measures to Project and Promote the Diversity of Cultural Expressions in the Framework of the UNESCO Convention, C(2012) 3186 final, de 23 de mayo de 2012.

43 Primer informe sobre la consideración de los aspectos culturales. COM (96) 160 final, de 17 de abril de 1996.

${ }^{44}$ Quadrennial Report on behalf of the European Union on Measures to Project and Promote the Diversity of Cultural Expressions in the Framework of the 2005 UNESCO Convention, 6 . 
nes de apoyo, coordinación o complemento, de acuerdo con el principio de subsidiariedad. La Unión puede pues actuar para apoyar y complementar la acción de los Estados, pero nunca en detrimento de la misma. Esta disposición ha sido modificada en una ocasión tras la entrada en vigor de la Convención de la UNESCO, con motivo del Tratado de Lisboa. Dicha reforma hizo posible la adopción por mayoría cualificada de las medidas de fomento de la cultura, eliminando la unanimidad que había prevalecido hasta entonces ${ }^{45}$.

Conviene mencionar que el énfasis en la diversidad del art.167 TFUE es previo a la firma de la Convención. El párrafo primero dispone que la Unión contribuirá al florecimiento de las culturas de los Estados miembros «dentro del respeto de su diversidad nacional y regional, poniendo de relieve al mismo tiempo el patrimonio cultural común», y el párrafo cuarto establece que la Unión tendrá en cuenta los aspectos culturales en su actuación en virtud de otras disposiciones del presente Tratado, a lo que la reforma de Ámsterdam añadió: «en particular a fin de respetar y fomentar la diversidad de sus culturas». Ello pone de manifiesto la continuidad del énfasis europeo en la diversidad cultural, antes y después de la Convención.

En segundo lugar, es preciso destacar la Agenda Europea de la Cultura, el documento que recoge, más allá de lo dispuesto por el art. 167 TFUE, los objetivos y prioridades de la política cultural de la Unión. Si bien las instituciones europeas comenzaron a financiar acciones culturales desde los años setenta, y las dotaron de una base jurídica firme con ocasión de la reforma de Maastricht, llama la atención el hecho de que hasta quince años después la Unión no realizase una reflexión sistemática sobre su relación con la cultura en sus múltiples dimensiones, definiendo sus prioridades y objetivos y proponiendo un nuevo método de trabajo. Como la propia Comisión Europea ha señalado ${ }^{46}$, fue a raíz de la Convención de la UNESCO cuando la Unión dio el paso más relevante en la definición de su política cultural al adoptar en 2007 la Agenda Europea para la Cultura.

La Agenda establecía tres grupos de objetivos. En primer lugar, proponía fomentar la diversidad cultural y el diálogo intercultural. Para ello, hacía referencia especial al fomento de la movilidad de artistas y profesio-

45 Belén Becerril Atienza, «La disposición cultural de los Tratados. Estudio del artículo 167 TFUE y de las modificaciones realizadas por el Tratado de Lisboa», en La UNESCO y la protección internacional de la cultura en el espacio iberoamericano, ed. por Cástor Diaz Barrado y Carlos Fernández de Liesa (Pamplona: Civitas, 2011): 391-423.

${ }^{46}$ Quadrennial Report on behalf of the European Union on Measures to Project and Promote the Diversity of Cultural Expressions in the Framework of the 2005 UNESCO Convention, 7. 
nales, de obras de arte, de trabajadores culturales... En segundo lugar, establecía el objetivo novedoso de promover la cultura como catalizador para la creatividad en el marco de la estrategia de Lisboa para el crecimiento y el empleo. En este sentido, se hacía énfasis en el creciente papel de las industrias culturales en el dinamismo y competitividad de la UE y se proponían objetivos más concretos como reforzar las capacidades organizativas del sector cultural y desarrollar asociaciones eficaces entre el sector cultural y otros, como la investigación o el turismo, para aumentar el impacto de las inversiones en la cultura. En tercer y último lugar, la Agenda establecía el objetivo de reforzar la dimensión cultural como elemento de las relaciones exteriores de la Unión. Para ello se proponía integrar sistemáticamente la cultura en todas las políticas con una dimensión exterior y promover los intercambios culturales con terceros países así como el acceso a los mercados mundiales de los bienes y servicios de los países en desarrollo. Tanto el énfasis prioritario en la diversidad cultural como el fortalecimiento de la dimensión cultural en la política exterior de la Unión, con especial mención de los países en desarrollo, ponían de manifiesto el alineamiento de la Agenda con la Convención.

Otra de las novedades de la Agenda se refería a los nuevos métodos de trabajo. Se consideraba llegado el momento de que los Estados diesen un paso más en la cooperación cultural y utilizasen el método abierto de coordinación, un marco intergubernamental no vinculante en el que los Estados tratan de acordar objetivos, seguir sus progresos e intercambiar buenas prácticas. En el marco de este método se definieron cinco prioridades en el Plan de Trabajo para el período 2008-2010 ${ }^{47}$, entre las que se encontraba la promoción y aplicación de la Convención de la Diversidad. Esta prioridad se ha mantenido como una constante, recogiéndose de nuevo en el Plan de Trabajo 2011-2014 ${ }^{48}$ y en el de 2015-2018 ${ }^{49}$, que se está desarrollando en la actualidad. Por último, la Agenda marcó también el inicio de un diálogo

47 Conclusiones del Consejo y de los Representantes de los Gobiernos de los Estados miembros, reunidos en el seno del Consejo, sobre el plan de trabajo en materia cultura (20082010), DO C 143 de 10.6.2008.

48 Conclusiones del Consejo y de los Representantes de los Gobiernos de los Estados miembros reunidos en el seno del Consejo, sobre el plan de trabajo en materia de cultura (2011-2014), DO C 325 de 2.12.2010. Las seis prioridades específicas se alineaban con las del plan previo. En aquel se subrayaba la promoción de la Convención UNESCO, mientras que ahora se destacaba «La cultura en las relaciones internacionales», dentro de la que a su vez se contempla la promoción de la Convención.

49 Conclusiones del Consejo y de los Representantes de los Gobiernos de los Estados miembros, reunidos en el seno del Consejo, sobre el Plan de trabajo en materia de cultura (2015-2018) DO C 463 de 23.12. 2014. La prioridad D se refería a la Promoción de la diversidad cultural, cultura en las relaciones exteriores de la UE y movilidad, y en primer lugar, a la Convención de la UNESCO. 
más estructurado con el sector cultural. A este respecto, el artículo 11 de la Convención disponía que las Partes fomentarían la participación activa de la sociedad civil.

\section{Los programas de apoyo al sector cultural}

Tras el impulso que supuso la entrada en vigor, con el Tratado de Maastricht, de la nueva disposición cultural, los programas de los años noventa, Calidoscopio, Ariane y Rafael $^{50}$ reorganizaron la acción cultural en torno a tres líneas: apoyo a las actividades artísticas y culturales, apoyo al libro y la lectura, incluida la traducción, y apoyo a la conservación del patrimonio. Estos tres ámbitos de actuación se consolidarían más tarde con el programa Cultura $2000^{51}$ que permitiría al fin contar con un planteamiento global, un instrumento financiero único y una dotación de 236,5 millones de euros para el período comprendido desde el inicio de 2000 hasta el fin de $2006^{52}$. Las líneas desarrolladas con esos programas se mantendrían también, en gran medida, tras la entrada en vigor de la Convención de la UNESCO, con los nuevos programas culturales. El programa Cultura (2007-2013) ${ }^{53}$, aprobado tras la firma de la Convención pero antes de su entrada en vigor, constituiría de nuevo un instrumento único de financiación y programación para la cooperación cultural, con una dotación de 400 millones de euros.

La promoción de la diversidad cultural sería un elemento fundamental del nuevo programa Cultura, a pesar de lo cual debe notarse que la Decisión no se refiere expresamente a la Convención. También destaca el énfasis del nuevo programa en la ciudadanía europea. En unos años difíciles para el proyecto de integración, el programa vinculaba la acción cultural al concepto de la ciudadanía, estableciendo como objetivo general «impulsar un espacio cultural compartido por los europeos y basado en una herencia

50 Decisión del Parlamento Europeo y el Consejo 719/96, por la que se aprueba el programa de apoyo a las actividades artísticas y culturales de dimensión europea, DO L 99 de 20.4.96, 20. Decisión del Parlamento Europeo y el Consejo 2085/97, DO L 291 de 24.10.97, 19. Decisión del Parlamento Europeo y el Consejo 2228/97, DO L 305 de 8.11.97, 31 .

${ }^{51}$ El programa Cultura 2000 fue aprobado mediante la Decisión 508/2000/CE del Parlamento Europeo y del Consejo, DO L 63 de 10. 3. 2000, 1.

${ }^{52}$ Se estableció una dotación de 167 millones de euros para el período comprendido entre el 1 de enero de 2000 y el 31 de diciembre de 2004. Posteriormente fue prorrogado a los años 2005 y 2006 sin modificarse su contenido, estableciéndose una dotación financiera global de 236,5 millones de euros para la totalidad del programa. Decisión de 31 de marzo de 2004 n. ${ }^{\circ}$ 508/2000/CE, DO L 99 de 3.4.2004.

53 Decisión n. ${ }^{\circ}$ 1855/2006/CE del Parlamento Europeo y del Consejo, de 12 de diciembre de 2006, por la que se establece el programa Cultura (2007-2013). 
cultural común, mediante el desarrollo de una cooperación cultural entre creadores, agentes culturales e instituciones culturales de los países participantes en el programa, con el fin de favorecer el surgimiento de una ciudadanía europea» (art.3). A ello seguirían tres objetivos específicos: promover la movilidad transnacional de los agentes culturales, fomentar la circulación transnacional de obras y productos artísticos y culturales y favorecer el diálogo intercultural.

Para alcanzarlos, la mayor parte del presupuesto se destinaría a financiar acciones culturales: proyectos plurianuales de cooperación, con la participación de operadores culturales de al menos seis Estados; acciones de cooperación, con especial énfasis en la creatividad y la innovación, y operadores culturales de al menos tres países participantes; y por último, acciones especiales, de escala y alcance importantes, que contribuyesen a tomar conciencia sobre la diversidad cultural y la pertenencia a una misma comunidad. Las Capitales Europeas de la Cultura, una de las iniciativas de más éxito de la acción cultural europea o la concesión de los premios al talento artístico, estarían financiadas por esta categoría, que incluiría también nuevas iniciativas como el Sello del Patrimonio Europeo.

Globalmente, podríamos destacar que el planteamiento del nuevo programa Cultura mantenía la continuidad respecto al pasado. Merece la pena especialmente destacar el énfasis en la movilidad transnacional de artistas y profesionales de la cultura, así como en la movilidad de obras de arte ${ }^{54}$. La Comisión ha estimado que se han beneficiado del programa anualmente cerca de 20.000 artistas y profesionales de la cultura que han podido fortalecer la dimensión internacional de sus carreras, así como 1.000 organizaciones culturales, que han podido participar en redes y proyectos internacionales $^{55}$. Cabe señalar también el énfasis en la cooperación con terceros Estados. El programa estaba abierto a la participación de diez Estados europeos, al margen de los miembros, y preveía la posibilidad de cooperación para terceros países con acuerdos bilaterales que contuviesen cláusulas culturales. De esta manera, se han financiado proyectos en China, India, Brasil, México y en los países de la Vecindad Europea, estableciéndose en este caso la condición de haber ratificado la Convención.

54 Interim Evaluation of the Culture Programme 2007-13. Final Report. ECORYS UK on behalf of the European Commission DG Education and Culture. Acceso el 10 de noviembre de 2016:

http://ec.europa.eu/dgs/education_culture/more_info/evaluations/docs/culture/ culture2010_en.pdf

55 Quadrennial Report on behalf of the European Union on Measures to Project and Promote the Diversity of Cultural Expressions in the Framework of the 2005 UNESCO Convention: 9 . 
A pesar de las fortalezas del programa, como su continuidad y coherencia, y el esfuerzo por mejorar su apertura y visibilidad, no podemos evitar señalar la prudencia del planteamiento con el que la Unión ha abordado la cultura y su limitada ambición, no sólo en lo que respecta a su dotación económica. Si observamos la magnitud y el impacto de la acción cultural en relación con otras políticas europeas, lo cierto es que, más allá de la retórica, el esfuerzo que con este programa se destinaba a la cultura era aún muy limitado. Destaca pues la notable distancia entre su verdadera dimensión y los ambiciosos objetivos sobre la construcción de una ciudadanía europea.

Al margen del programa Cultura había quedado la industria audiovisual, que estaba cubierta por el programa MEDIA (2007-2013) ${ }^{56}$. Este se había desarrollado para apoyar al cine y al sector audiovisual y había contado con 750 millones de euros. Entre sus objetivos se destacaba en primer lugar, en línea con la Convención, «preservar y realzar la diversidad cultural y lingüística y el patrimonio cinematográfico y audiovisual europeos, garantizar que el público pueda acceder a ellos, y favorecer el diálogo intercultural». También se caracterizaba por su posible apertura a Estados europeos no miembros, que de acuerdo con su artículo 8 podía someterse a un examen de compatibilidad de su legislación nacional con el acervo comunitario, examen que incluiría ${ }^{57}$ la ratificación de la Convención. A este programa se sumaría más tarde MEDIA Mundus (2011-2013) ${ }^{58}$ con el fin de promover la cooperación audiovisual entre los profesionales europeos y sus homólogos de terceros países y una dotación de 15 millones de euros. Este se refería expresamente en su exposición de motivos a la Convención y a su artículo 12 e), en virtud del cual las Partes procurarán fomentar la firma de acuerdos de coproducción y codistribución.

El último de los programas culturales emprendido por la Unión ha sido Europa Creativa $^{59}$, para el período 2014-2020. Este agrupa las acciones an-

${ }^{56}$ Decisión n ${ }^{\circ}$ 1718/2006/CE del Parlamento Europeo y del Consejo de 15 de noviembre de 2006 relativa a la aplicación de un programa de apoyo al sector audiovisual (Media 2007) DO 1327/12 de 24.11.2006.

57 Quadrennial Report on behalf of the European Union on Measures to Project and Promote the Diversity of Cultural Expressions in the Framework of the 2005 UNESCO Convention, 10. MEDIA también se abriría a otros Estados que hubiesen concluido acuerdos de asociación o de cooperación con la Unión que contengan cláusulas relativas al sector audiovisual y «compartiesen una aproximación común a la diversidad cultural».

58 Decisión n. ${ }^{\circ}$ 1041/2009/CE del Parlamento Europeo y del Consejo de 21 de octubre de 2009 por la que se establece un programa de cooperación audiovisual con profesionales de terceros países DO L 288/10 de 4.11.2009.

59 Reglamento No 1295/2013 del Parlamento Europeo y del Consejo de 11 de diciembre de 2013 por el que se establece el Programa Europa Creativa (2014 a 2020). 
tes cubiertas por el programa Cultura junto con MEDIA y MEDIA Mundus, incrementando el presupuesto global en un 9\%, y destinando al sector 1.460 millones en un período de siete años. El nuevo programa, a diferencia del anterior, menciona repetidamente en la Convención desde el primer párrafo de su exposición de motivos. A continuación, establece dos objetivos generales, «salvaguardar, desarrollar y promover la diversidad cultural y lingüística europea y promover el patrimonio cultural europeo», y «reforzar la competitividad de los sectores cultural y creativo europeos, en particular el sector audiovisual, con vistas a promover un crecimiento inteligente, sostenible e integrador».

Europa Creativa da así seguimiento a una pauta que viene poniéndose de manifiesto desde la Agenda, como es el énfasis especial en las industrias culturales y creativas y en su potencial para contribuir a la competitividad europea $^{60}$. El nuevo programa incluye iniciativas de los anteriores programas Cultura, como el apoyo a redes y proyectos de cooperación transfronteriza, la traducción y promoción de obras literarias, las Capitales Europeas de la Cultura, el Sello de Patrimonio Europeo, los premios europeos de la literatura, arquitectura, protección del patrimonio... Pero ahora, el programa contiene también las iniciativas para apoyar el sector audiovisual, incluyendo la distribución y comercialización de obras audiovisuales, los festivales de cine europeo, los fondos para la coproducción internacional de películas...

De este modo, el nuevo programa concentra el apoyo a la cultura y a la industria audiovisual, a la vez que enfatiza y apoya su contribución al crecimiento económico, motivo por el cual se ha hablado de un «giro industrial» en la política cultural ${ }^{61}$. Frente a conceptos complejos como la ciudadanía europea o la diversidad cultural, a la hora de evaluar los proyectos se atenderá en particular a los indicadores económicos como su impacto en el empleo, el número de personas que acceden a las obras o al número de entradas de películas europeas en el mundo ${ }^{62}$. El objetivo de promover la diversidad cultural orienta el programa, pero en su implementación se refuerza el apoyo a las industrias culturales en base a criterios cuantitativos.

${ }^{60}$ La Comisión había lanzado una consulta y publicado sus resultados en un Libro Verde en el que proponía integrar mejor estas industrias en la estrategia Europa 2020, COM (2010) 183 final - . Como continuación, la Comisión preparó el documento «Liberar el potencial de las industrias culturales y creativas», SEC (2011) 399 final.

${ }^{61}$ Rachael Craufurd Smith, »The Cultural logic of Economic Integration», en Cultural Governance and the European Union: Protecting and Promoting Cultural Diversity in Europe, ed. por Evangelia Psychogiopoulou (Palgrave Macmillan, 2015): 19.

62 Véase artículo 18 del Reglamento de Europa Creativa. 


\section{La regulación de servicios audiovisuales}

\section{a. La directiva de servicios de comunicación audiovisual}

Tras la entrada en vigor de la Convención, en el año 2010 se aprobó el acto más relevante de la política audiovisual de la Unión, la directiva de servicios de comunicación audiovisual ${ }^{63}$. Esta venía a sustituir la directiva Televisión Sin Fronteras, aprobada a finales de los ochenta y revisada ya en varias ocasiones $^{64}$, que se había adoptado con el propósito de armonizar las legislaciones de los Estados miembros para evitar que impidiesen la recepción en su territorio de emisiones procedentes de sus socios comunitarios. La directiva Televisión Sin Fronteras regulaba diversos aspectos, como la publicidad y el patrocinio, la protección de menores o el derecho de réplica y también trataba de promocionar la industria audiovisual europea. Para ello, requería en primer lugar, que siempre que fuese posible, los organismos de radiodifusión televisiva reservasen una proporción mayoritaria de su tiempo de difusión a obras europeas (art.4), y en segundo lugar, que siempre que fuese posible, reservasen al menos el $10 \%$ de su tiempo de emisión o el $10 \%$ de su presupuesto de programación a obras europeas de productores independientes (art.5). Estas disposiciones estaban redactadas en términos que ponían en duda su obligatoriedad. No obstante, fueron objeto de críticas ${ }^{65}$ y motivo de un tenso desencuentro con los Estados Unidos que en la Ronda de Uruguay trataron sin éxito de que los europeos se comprometiesen a su supresión.

La nueva directiva de 2010 respondió a un esfuerzo de adaptación a los cambios estructurales en el sector, la difusión de las tecnologías de la información y de las comunicaciones y la evolución tecnológica. A diferencia de Televisión sin Fronteras, la nueva directiva no se aplicaría sólo a la radiodifusión televisiva, sino a los servicios de medios audiovisuales, con el fin de que estos puedan circular libremente en el mercado único europeo con independencia de su formato de emisión - televisión tradicional, vídeo a la carta, internet...-. En lo que respecta a la Convención, puede afirmarse que su letra y su espíritu están bien presentes en la nueva directiva. Esta se refiere repetidamente al respeto de la diversidad cultural y lingüística y cita

${ }^{63}$ Directiva 2010/13/UE del Parlamento Europeo y del Consejo de 10 de marzo de 2010 sobre la coordinación de determinadas disposiciones legales, reglamentarias y administrativas de los Estados miembros relativas a la prestación de servicios de comunicación audiovisual.

${ }^{64}$ Directiva 89/552/CEE del Consejo (DO L 298 de 17.10.1989, p. 23). Modificada por la directiva 97/36/CE del Parlamento Europeo y del Consejo (DO L 202 de 30.7.1997, p. 60) y la directiva 2007/65/CE del Parlamento Europeo y del Consejo (DO L 332 de 18.12.2007, p. 27).

65 Santiago Muñoz Machado criticó el «dirigismo cultural» y la injerencia en las competencias culturales de los Estados en Público y privado en el mercado europeo de la televisión (Madrid: Civitas, 1991): 68. 
la Convención para recordar «que las actividades, los bienes y los servicios culturales son de índole a la vez económica y cultural, porque son portadores de identidades, valores y significados, y por consiguiente no deben tratarse como si solo tuviesen un valor comercial».

En cuanto a la promoción de obras europeas e independientes, la directiva mantiene lo dispuesto en Televisión Sin Fronteras. Establece así que los Estados miembros velarán para que los organismos de radiodifusión televisiva reserven para obras europeas una proporción mayoritaria de su tiempo de difusión, con exclusión del tiempo dedicado a las informaciones, manifestaciones deportivas, juegos, publicidad, servicios de teletexto y televenta (art.16). Por otra parte, dispone que los organismos de radiodifusión televisiva deberán consagrar al menos el $10 \%$ de su tiempo en antena, o el $10 \%$ de su presupuesto de programación, a obras europeas realizadas por productores independientes (art.17). En ambos casos, se dice que tal cosa se hará «siempre que sea posible», dejando su obligatoriedad de nuevo en entredicho. En lo que respecta a los servicios de comunicación audiovisual a la carta, en los que no cabe requerir una proporción mayoritaria de tiempo de difusión ya que los consumidores pueden elegir las obras que visualizan, la directiva se limita a disponer que los Estados velarán por que los prestadores de servicios promuevan la producción de y el acceso a las obras europeas, pudiendo «contribuir a la financiación de la producción de obras europeas o reservar una parte y/o un espacio importante a tales obras en sus catálogos de programas» (art.13). En la transposición de la directiva, varios Estados han requerido reservar un porcentaje de las obras del catálogo a las europeas.

Así pues, puede afirmarse que uno de los elementos cruciales de esta directiva, piedra angular del sector audiovisual, es la promoción de obras europeas e independientes, un objetivo aparentemente alineado con la Convención. No obstante, conviene notar que este esfuerzo es en realidad muy anterior a la misma. Además, la directiva promociona las obras europeas, no la diversidad cultural en sí misma, y lo hace con base a unos criterios que tienen que ver el origen de la obra - residencia de sus autores y trabajadores, realización o supervisión de un productor establecido en un Estado europeo, contribución mayoritaria de coproductores europeos...-, más que con su contribución a la diversidad o su calidad. Por eso, algunos han señalado que la preocupación de la Unión parece más centrada en sus aspectos industriales o empresariales que en los culturales ${ }^{66}$. No obstante, teniendo en cuenta el contexto de la Convención, su énfasis en el sector audiovisual y su objetivo prioritario de afirmar el derecho de las Partes para adoptar

${ }^{66}$ En el mismo sentido, Mira Burri, «Business as Usual? An Appraisal of the Implementation of the UNESCO Convention on Cultural Diversity into EU's Media Law and Policies»: 813 . 
medidas destinadas a promover la diversidad de las expresiones culturales, puede afirmarse que al promocionar las obras europeas e independientes la directiva contribuye a la implementación de la Convención y esta a su vez refuerza la legitimidad de la norma europea, a pesar de su carácter proteccionista.

\section{b. La sentencia UTECA}

La directiva permite también a los Estados miembros aplicar normas más estrictas o detalladas, en los ámbitos coordinados por la misma, a los prestadores del servicio de comunicación bajo su jurisdicción, siempre que estas normas sean conformes con los principios generales del Derecho de la Unión. Esta cuestión se planteó en el mencionado asunto UTECA (Unión de Televisiones Comerciales Asociadas), en el que se cuestionaba la compatibilidad de la Directiva Televisión sin Fronteras con una norma española que obligaba a los operadores de televisión a destinar el $5 \%$ de sus ingresos de explotación a la financiación anticipada de películas europeas y, más concretamente, el $60 \%$ de dicho $5 \%$ a obras cuya lengua original sea cualquiera de las oficiales en España. El gobierno español alegaba que la disposición cuestionada se basaba en «razones culturales de defensa del multilingüismo español», cosa que el Tribunal de Justicia aceptó reconociendo que el objetivo de «defender y promover una o varias de sus lenguas oficiales» constituye una razón imperiosa de interés general capaz de justificar la restricción al principio de libre circulación, siempre que sea adecuada para garantizar la realización del objetivo perseguido, y no vaya más allá de lo necesario para lograrlo, es decir, que sea proporcional.

Conviene señalar que la sentencia UTECA, en la que el Tribunal de Justicia citaba la Convención por vez primera, es un caso especialmente relevante que manifiesta una cierta flexibilización en la jurisprudencia sobre el mercado interior y las políticas culturales nacionales. Esta flexibilización ya se había vislumbrado en la sentencia de 2007 United Pan Europe ${ }^{67}$, precisamente el año de entrada en vigor de la Convención, en la que el Tribunal se había pronunciado sobre una norma que restringía la libre prestación de servicios afirmando expresa y rotundamente que «una política cultural puede constituir una razón imperiosa de interés general que justifique una restricción ${ }^{68}$.

67 Sentencia de 13 de diciembre de 2007, United Pan Europe Communications Belgium, asunto C-250/06.

68 Desde mediados de los ochenta el Tribunal venía aceptando motivos culturales - como el fomento de las obras cinematográficas o la promoción de la lengua nacional- 
En primer lugar, en UTECA el Tribunal descartó las alegaciones de la Comisión en virtud de las cuales la norma no establecía criterios objetivos y verificables para garantizar que el régimen de financiación sólo se aplicase a películas que pudiesen ser consideradas «productos culturales». El Tribunal citó el párrafo décimo quinto de la Convención en virtud del cual «la diversidad lingüística es un elemento fundamental de la diversidad cultural» y consideró que la lengua y la cultura están intrínsecamente vinculadas de modo que: «no pude afirmarse que el objetivo perseguido por un Estado miembro, de promover una o varias de sus lenguas oficiales deba ir acompañado necesariamente de otros criterios culturales para que pueda justificar una restricción de una de las libertades fundamentales garantizadas por el Tratado».

En segundo lugar, en UTECA el Tribunal no hizo referencia alguna a la necesidad de que la norma fuese indistintamente aplicable para poder ser justificada en una razón imperiosa de interés general, señalando que la ventaja conferida a las empresas que trabajasen en esa lengua resultaba inherente al objetivo perseguido. De esta manera, el Tribunal dejaba atrás su jurisprudencia en virtud de la cual las razones imperiosas de interés general sólo podían justificar normas indistintamente aplicables. Respondía así a los que habían solicitado «una cierta agilización de la jurisprudencia», sugiriendo que el carácter discriminatorio podría ser inherente a las normas de política cultural ${ }^{69}$. Poco después, en $L I B R O^{70}$, en relación a una normativa austriaca que prohibía a los importadores de libros en alemán fijar libremente el precio de venta, el Tribunal confirmaría esta apertura, aceptando la posibilidad de justificar una norma que por razones culturales impone un trato desigual.

Como se ha mencionado, en este asunto, el Tribunal citaba la Convención por vez primera. Seguía así la estela marcada por la Abogada General Kokott que al precisar el marco jurídico aplicable se había referido dilatadamente a la Convención (apartados 13-19), y había recordado que en virtud de su artículo 20.1.b) la Comunidad y los Estados miembros se han comprometido a tener en cuenta las disposiciones de la Convención cuando interpreten y apliquen otros tratados, es decir, también al interpretar y aplicar el Tratado CE (apartado 99). En esta línea, la sentencia del

como intereses susceptibles de justificar una restricción. No obstante, se resistía aún a configurar de forma expresa el interés cultural, o la política cultural, de un modo general, como un nuevo motivo de excepción.

69 Conclusiones del Abogado General Van Gerven. Sentencia de 4.5.1993, Fedicine, asunto C-17/92.

70 Sentencia de 30.4.2009, Fachverband der Buch- und Medienwirtschaft y LIBRO, asunto C-531/07. 
Tribunal citaba la Convención y «la tenía en cuenta» a la hora de interpretar el Derecho de la Unión, en particular, para justificar el vínculo existente entre la lengua y la cultura. No obstante, a diferencia de la Abogada General, no afirmaba expresamente su consideración a efectos interpretativos, y en realidad, a la hora de justificar la restricción al principio de libre circulación por motivos lingüísticos, recurría a su jurisprudencia anterior, que a estos efectos era suficiente. A pesar de ello el Tribunal, lejos de invocar el art.20.2. o su Declaración unilateral ${ }^{71}$ para descartar la aplicación de la Convención en el ordenamiento de la Unión, abría la puerta, con prudencia, al reconocimiento de sus efectos interpretativos. Por último cabe mencionar que también la Comisión Europea mencionó explícitamente la Convención en 2012 en sus decisiones sobre dos ayudas concedidas por España para la publicación de revistas culturales y de literatura en el País Vasco ${ }^{72}$.

\section{Conclusiones}

La Unión Europea jugó un papel novedoso y decisivo en la negociación de la Convención de la Diversidad en el seno de la UNESCO, contribuyendo a la consolidación del concepto mismo de la diversidad cultural en la escena internacional y enfatizando el principio de soberanía en virtud del cual los Estados tienen el derecho de adoptar las medidas y políticas que estimen necesarias para proteger y promover la diversidad de las expresiones culturales en sus respectivos territorios.

Por este motivo, y porque hizo de su promoción un objetivo prioritario, puede decirse que la Unión Europea tiene una especial responsabilidad en la implementación de la Convención. Tanto más si se considera que, como se ha visto, dada la muy limitada obligatoriedad de sus disposiciones y el hecho de que estas no ponen en cuestión las obligaciones que para las Partes emanan de otros tratados internacionales, la eficacia de la Convención depende en gran medida de la voluntad de las Partes para implementarla dentro de sus fronteras.

Diez años tras su entrada en vigor, puede afirmarse que el espíritu y la letra de la Convención están bien presentes en el marco político y regulatorio de la política cultural y audiovisual de la Unión. Como se ha visto, con

71 Véase la mencionada Declaración unilateral de la Comunidad en el anexo 2 de la De-

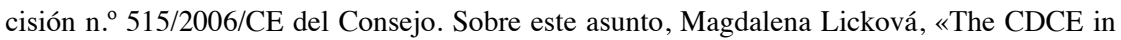
the European Union - a mixed agreement and its judicial application»: 229.

${ }^{72}$ SA.34138/2012/N - Ayuda para publicación de revistas culturales, SA.34168 2012/N - Ayuda para la publicación de literatura en el País Vasco. 
algunas excepciones, la Convención es una referencia obligada en los actos europeos en la materia y la protección y promoción de la diversidad cultural es un objetivo prioritario. Es preciso no obstante notar que este énfasis en la diversidad no es nuevo y era una constante de la acción cultural europea desde sus primeros pasos, mucho antes del año 2007. En realidad, la Convención proyecta en la escena internacional un concepto de diversidad cultural cuya promoción ya ocupaba el lugar prioritario entre los objetivos de la acción cultural de la Unión, como muestra la letra de la disposición cultural de los Tratados (art.167 TFUE). No obstante, sí puede afirmarse que tras la Convención la Unión dio un paso más en su discurso programático con la aprobación de la Agenda Europea de la Cultura, en la que realizaba un ejercicio sistemático de definición de sus objetivos y prioridades. Tras décadas en las que la acción cultural de la Unión constituía poco más que una serie de iniciativas aisladas, con la Convención y con la Agenda la Unión realizaba el esfuerzo programático pendiente para la articulación de una verdadera política cultural.

En cuanto a las iniciativas adoptadas para la implementación de la Convención en el marco de la política cultural y audiovisual de la Unión, cabe de nuevo destacar la continuidad. Los programas de promoción cultural y audiovisual, Cultura, Media y después, Europa Creativa, son en realidad una prolongación de los anteriores. De ellos cabe destacar particularmente el énfasis en la movilidad de Cultura 2007, un objetivo alineado con la Convención pero no novedoso, y un cierto giro industrial en el programa Europa Creativa. También, un esfuerzo creciente por la consideración de criterios cuantitativos, lejos de la retórica, a la hora de evaluar la contribución de los proyectos culturales y un aumento de la financiación, que sigue no obstante siendo muy limitada. Por otra parte, la continuidad es también la nota más destacada, a este respecto, en la regulación del sector audiovisual. Las cuotas reservadas a obras europeas e independientes se han mantenido inalteradas desde los años noventa.

Puede pues concluirse que frente a algunas iniciativas novedosas adoptadas por la Unión para la implementación de la Convención en su dimensión externa - como la firma de los mencionados y controvertidos protocolos culturales - , en su dimensión interna ha primado la continuidad. La Convención parece haber tenido más un efecto legitimador de las medidas existentes, que impulsor de nuevas iniciativas para la protección y promoción de las expresiones culturales ${ }^{73}$.

73 A la misma conclusión llega, en el marco de la política audivisual europea, María Trinidad García Leiva, «Política audiovisual europea y diversidad cultural en la era digital», 221. 
Por lo demás, conviene señalar que el impacto que la política cultural de la Unión puede de hecho ejercer en la promoción de la diversidad cultural sigue siendo limitado, como limitado es el esfuerzo financiero que la Unión dedica a esta política. Por este motivo es de especial interés atender, junto a la política de promoción cultural emprendida desde las instituciones, a la relación del mercado interior con las normas nacionales de política cultural. Desde esta perspectiva se ha destacado el asunto UTECA, en el que el Tribunal de Justicia citaba por vez primera la Convención y lejos de descartar su eficacia, abría la puerta al reconocimiento de sus efectos interpretativos. Conviene también notar que, precisamente en esta sentencia, el Tribunal daba un paso relevante en la flexibilización de su jurisprudencia, permitiendo la justificación de normas discriminatorias por exigencias imperativas de política cultural. La Convención ha contribuido pues a acentuar, en este asunto, el énfasis en la diversidad cultural, afectando a una jurisprudencia definida cuidadosamente a lo largo de los años en torno a los principios de eficacia y proporcionalidad. Para terminar, cabe también mencionar que esta larga experiencia de la Unión en la búsqueda de un equilibrio adecuado entre el mercado y la cultura podría ser de utilidad en el futuro si en efecto la Convención marca sólo el inicio de un camino hacia la mayor consideración de la dimensión cultural de los bienes y servicios en el comercio internacional.

\section{Bibliografía}

Balassa, Carol. «The Impact of the US Position in the Trade and Culture Debate». En The UNESCO Convention on the Diversity of Cultural Expressions, editado por Toshiyuki Kono y Steven Van Uystel, 71-94. Cambridge: Intersentia, 2012.

Baltá, Jordi. «Aplicación en la Unión Europea de la Convención de la UNESCO de 2005 sobre la Protección y Promoción de la Diversidad de las Expresiones Culturales», estudio solicitado por la Comisión de Cultura y Educación del Parlamento Europeo, Bruselas 2010.

Becerril Atienza, Belén. «La disposición cultural de los Tratados. Estudio del artículo 167 TFUE y de las modificaciones realizadas por el Tratado de Lisboa». En La UNESCO y la protección internacional de la cultura en el espacio iberoamericano, editado por Cástor Diaz Barrado y Carlos Fernández de Liesa, 391-423. Pamplona: Civitas, 2011.

Becerril Atienza, Belén: Hacia una política Cultural de la Unión Europea. Cizur Menor: Thomson Reuters Aranzadi, 2015.

Becerril Atienza, Belén. «La política cultural de la Unión Europea». En Tratado de Derecho y Políticas de la Unión Europea, tomo VII, editado por José María Beneyto, Jerónimo Maíllo y Belén Becerril, 1003-1056. Cizur Menor: Thomson Reuters Aranzadi, 2016. 
Bernier, Ivan. "The Convention on the Protection and the Promotion of the Diversity of Cultural Expressions, a Cultural Analysis». En The UNESCO Convention on the Diversity of Cultural Expressions, ed. por Toshiyuki Kono y Steven Van Uystel. 95-123. Cambridge: Intersentia, 2012.

Burri, Mira. «Business as Usual? An Appraisal of the Implementation of the UNESCO Convention on Cultural Diversity into EU's Media Law and Policies», European Law Review n. ${ }^{\circ} 3$ (2003): 802-825.

Burri, Mira. «Keeping promises: Implementing the UNESCO Convention on Cultural Diversity into EU's Internal Policies», NCCR Trade Regulation Working Paper No. 2010/17, University of Bern Law School - World Trade Institute.

Burri, Mira. «Cultural Diversity as a concept of Global Law: origins, evolution and prospects», Diversity, n. ${ }^{\circ} 2$ (2010): 1059-1084.

Cavicchioli, Lucia. «The European Community at UNESCO: an exceptionally active observer?». En The United Nations and the European Union: an ever stronger partnership, editado por Jan Wouters, Frank Hoffmeister y Tom Ruys, 135-154. The Hague: Asser, 2006.

Craufurd Smith, Rachael. «The UNESCO Convention on the Protection and Promotion of the Diversity of Cultural Expressions: Building a New World Information and Communication Order?», International Journal of Communication, n. ${ }^{\circ} 1$ (2007): 24-55.

Craufurd Smith, Rachael. «From Heritage Conservation to European Identity: Article 151 EC and the Multi-faceted Nature of Community Cultural Policy», European Law Review, n. ${ }^{\circ} 1$ (2007): 48-69.

Craufurd Smith, Rachael. «The Evolution of Cultural Policy in the European Union». En The Evolution of EU Law, editado por Paul Craig and Gráinne de Burca, 869-894. Oxford: Oxford University Press, 2011.

Craufurd Smith, Rachael. »The Cultural logic of Economic Integration». En Cultural Governance and the European Union: Protecting and Promoting Cultural Diversity in Europe, editado por Evangelia Psychogiopoulou, 7-23. Palgrave Macmillan, 2015.

Crusafon, Carmina. «Audiovisual europeo, diversidad cultural y mercado global: análisis de las actuaciones exteriores de la política audiovisual de la Unión Europea», Ámbitos, n. ${ }^{\circ} 19$ (2010): 243-263.

Crusafon, Carmina. «EU Cultural Cooperation with third countries: The Cases of Latin America and the Mediterranean». En Cultural Governance and the European Union: Protecting and Promoting Cultural Diversity in Europe, editado por Evangelia Psychogiopoulou, 225-235. Palgrave Macmillan, 2015.

Cunningham, Collete. «In Defense of Member State Culture: the Unrealized Potencial of Article 151.4 of the EC Treaty and the Consequences for EC Cultural Policy», Cornell International Law Journal, n. ${ }^{\circ} 34$ (2001): 119-163.

Ferri, Delia, «EU Participation in the UNESCO Convention on the Protection and Promotion of the Diversity of Cultural Expressions: Some Constitutional Remarks», European Diversity and Autonomy Papers, n. ${ }^{\circ} 3$ (2005): 1-34.

García Leiva, María Trinidad. «Política audiovisual europea y diversidad cultural en la era digital», Comunicación y sociedad, n. ${ }^{\circ} 27$ (2016): 221-241. 
Kono, Toshiyuki y Steven Van Uystel (eds). The UNESCO Convention on the Diversity of Cultural Expressions, A tale of Fragmentation in International Law. Cambridge: Intersentia, 2012.

Licková, Magdalena. «The CDCE in the European Union - a mixed agreement and its judicial application». En Cultural Diversity in International Law, The effectiveness of the UNESCO Convention on the Protection and Promotion of the Diversity of Cultural Expressions, editado por Lilian Richieri Hanania, 225237. Abingdon: Routledge, 2014.

Littoz-Monnet, Annabelle. The European Union and Culture. Between economic regulation and European cultural policy. Manchester and NY: Manchester University Press, 2007.

Loisen, Jan. «Mainstreaming Culture in EU External Relations through Protocols on Cultural Cooperation: Fostering or Faltering Cutural Diversity?». En The Palgrave Handbook of European Media Policy, editado por Karen Donders, Caroline Pawels y Jan Loisen, 509-525. Palgrave Macmillan, 2014.

Neuwirth, Rostam J. «The Convention on the Diversity of Cultural Expressions. A critical Analysis of the provisions». En The UNESCO Convention on the Diversity of Cultural Expressions, editado por Toshiyuki Kono y Steven Van Uystel, 229-272. Cambridge: Intersentia, 2012.

Jesús Prieto de Pedro. Excepción y diversidad cultural. Madrid: Alternativas, 2005. Psychogiopoulou, Evangelina. Integration of Cultural Considerations in European Union Law and Policies. Leiden, Boston: Martinus Nijhoff Publishers, 2007.

Psychogiopoulou, Evangelina. "The "Cultural" Criterion in the European Commission's Assessment of State Aids to the Audio-Visual Sector». Legal Issues of Economic Integration, n. 37 (2010): 273-291.

Psychogiopoulou, Evangelina. «The Convention on the Diversity of Cultural Expressions and the European Union». En The UNESCO Convention on the Diversity of Cultural Expressions, editado por Toshiyuki Kono y Steven Van Uystel, 365-394. Cambridge: Intersentia, 2012.

Psychogiopoulou, Evangelina. Cultural Governance and the European Union: Protecting and Promoting Cultural Diversity in Europe. Palgrave Macmillan, 2015.

Regourd, Serge. L'exception culturelle. Paris: Puf, 2003.

Richieri Hanania, Lilian (ed.). Cultural Diversity in International Law, The effectiveness of the UNESCO Convention on the Protection and Promotion of the Diversity of Cultural Expressions. Abingdon: Routledge, 2014.

\section{Sobre el autor}

Belén Becerril Atienza es profesora de Derecho de la Unión Europea de la Facultad de Derecho y Subdirectora del Instituto de Estudios Europeos de la Universidad CEU San Pablo. Doctora en Derecho por la Universidad CEU San Pablo (Premio Europa de tesis doctorales - Comunidad de Madrid) y Máster en Derecho Internacional por la Universidad de 
Lovaina (Gran Distinción), es autora de diversas publicaciones académicas sobre el proceso de integración europeo, en particular sobre su historia, sistema institucional, mercado interior y política cultural. Sus últimas publicaciones han sido: «La apuesta de los Estados Unidos por la unidad europea en el marco del Plan Marshall. El apoyo norteamericano y la obstrucción británica en el umbral de la integración europea (1947-1951)», Revista de Derecho Comunitario Europeo, 56, 2017, pp. 159-198; Winston Churchill. Europa Unida, Ediciones Encuentro, 2016; y Hacia una Política Cultural de la Unión Europea, Aranzadi, 2015. Ha sido asesora jurídica externa de miembros del Parlamento Europeo y del diputado Gabriel Cisneros durante la Convención que preparó la Constitución Europea. Ha realizado estancias e impartido clases en diversas Universidades europeas y de Estados Unidos como la Universidad de Sabanci en Estambul, Pazmany University en Budapest o William and Mary School of Law en Virginia. Ha participado en diversos proyectos de investigación financiados entre otros por el Ministerio de Educación y Ciencia, la Comisión Europea y el Parlamento Europeo. El presente estudio se enmarca dentro de la Red de Excelencia sobre «Los actuales desafíos del Derecho Internacional», del Plan Estatal de Investigación Científica y Técnica y de Innovación 20132016 (DER15-69273-RED).

\section{About the author}

Belén Becerril Atienza is Deputy Director of the Institute of European Studies at CEU San Pablo University. She holds a PhD in Law from Universidad CEU San Pablo (European Doctorate Thesis Award Community of Madrid) and a Masters in International Law from the University of Louvain (Great Distinction). She is the author of academic publications on the European integration process, in particular on its history, institutional system, internal market and cultural policy. Her latest publications have been: «La apuesta de los Estados Unidos por la unidad europea en el marco del Plan Marshall. El apoyo norteamericano y la obstrucción británica en el umbral de la integración europea (1947-1951)», Revista de Derecho Comunitario Europeo, 56, 2017, pp. 159-198; Winston Churchill. Europa Unida, Ediciones Encuentro, 2016; and Hacia una Política Cultural de la Unión Europea, Aranzadi, 2015. She has been external legal adviser to members of the European Parliament and of the Member Gabriel Cisneros during the Convention that drafted the European Constitutional treaty. She has been visiting scholar at several European and US universities such as Sabanci University in Istanbul, Pazmany University in Budapest or William and Mary School of Law in Virginia. 
She has participated in several research projects funded, among others, by the Ministry of Education and Science, the European Commission and the European Parliament. This study is part of the Network of Excellence on «Current Challenges of International Law», Plan Estatal de Investigación Científica y Técnica y de Innovación 2013-2016 (DER15-69273-RED). 


\section{Derechos de autor}

Los derechos de autor (para la distribución, comunicación pública, reproducción e inclusión en bases de datos de indexación y repositorios institucionales) de esta publicación (Cuadernos Europeos de Deusto, CED) pertenecen a la editorial Universidad de Deusto. El acceso al contenido digital de cualquier número de Cuadernos Europeos de Deusto es gratuito inmediatamente después de su publicación. Los trabajos podrán leerse, descargarse, copiar y difundir en cualquier medio sin fines comerciales y según lo previsto por la ley; sin la previa autorización de la Editorial (Universidad de Deusto) o el autor. Así mismo, los trabajos editados en CED pueden ser publicados con posterioridad en otros medios o revistas, siempre que el autor indique con claridad y en la primera nota a pie de página que el trabajo se publicó por primera vez en $C E D$, con indicación del número, año, páginas y DOI (si procede). Cualquier otro uso de su contenido en cualquier medio o formato, ahora conocido o desarrollado en el futuro, requiere el permiso previo por escrito del titular de los derechos de autor.

\section{Copyright}

Copyright (for distribution, public communication, reproduction and inclusion in indexation databases and institutional repositories) of this publication (Cuadernos Europeos de Deusto, CED) belongs to the publisher University of Deusto. Access to the digital content of any Issue of Cuadernos Europeos de Deusto is free upon its publication. The content can be read, downloaded, copied, and distributed freely in any medium only for non-commercial purposes and in accordance with any applicable copyright legislation, without prior permission from the copyright holder (University of Deusto) or the author. Thus, the content of $C E D$ can be subsequently published in other media or journals, as long as the author clearly indicates in the first footnote that the work was published in $C E D$ for the first time, indicating the Issue number, year, pages, and DOI (if applicable). Any other use of its content in any medium or format, now known or developed in the future, requires prior written permission of the copyright holder. 